\title{
Decreasing the risk of iatrogenic lymphoedema after axillary surgery: a threefold intervention
}

\author{
Sophie Bates, Rosemary Sedgwick
}

\begin{abstract}
Procedures such as venepuncture and cannulation can precipitate Breast Cancer Related Lymphoedema (BCRL) in patients who have undergone axillary surgery. We noted that procedures were inadvertently being performed on the side of surgery at our hospital, as susceptible patients were not clearly identifiable to staff.
\end{abstract}

An online anonymous staff survey at our hospital revealed that $26.9 \%$ of responders had seen procedures being performed on at risk arms in non-emergency settings. $83.3 \%$ of responders felt an intervention to allow easy identification of at risk arms would be useful.

Our intervention was threefold. Firstly, we created an 'At Risk Arm' alert on the computerised records system. Secondly, we produced a warning sign for each susceptible patient's bed. The signs are displayed above beds, and returned to notes for use if the patient is re-admitted. Thirdly, we informed GPs via discharge summaries of the need to perform procedures on the opposite side to surgery.

A second staff survey was conducted after implementation. $46.2 \%$ of responders felt that the new interventions would decrease the chance of patients developing BCRL. $61.5 \%$ felt that susceptible patients being more identifiable to staff would decrease the likelihood of procedures being performed on at risk arms.

Our project showed the importance of ensuring axillary surgery patients are clearly identifiable to hospital staff during admission. Further interventions could include wristbands or patient alert cards. This work could be extended to include primary care and outpatient settings.

\section{Problem}

Axillary surgery such as axillary node clearance or sentinel lymph node biopsy is a known risk factor for the development of lymphoedema. Royal College of Anaesthetist Guidelines for Breast Cancer Related Lymphoedema state that procedures such as venepuncture, blood pressure measurement and cannulation can precipitate the condition, and should thus be performed on the opposite side to surgery where possible.

We noted there was no effective system in place at our hospital, to allow staff to clearly identify patients who had had previous axillary surgery. Procedures were inadvertently being performed on the same side as surgery, potentially increasing risk of developing lymphoedema.

\section{Background}

Lymphoedema associated with breast cancer treatment is a significant health burden on both patients and NHS services. In a study of 228 patients with chronic lymphoedema, $27 \%$ had been admitted requiring IV antibiotics, with a mean length of stay of 12 days, the estimated cost being $£ 2300$ per admission. In addition, more than $80 \%$ had taken time off work and $50 \%$ reported a lower quality of life, due to pain or discomfort as a result of the condition (1).
The Royal College of Anaesthetist Guidelines for Breast Cancer Related Lymphoedema (BCRL) state that puncture wounds, including venepuncture have been identified as precipitating factors for the condition. Their recommendations suggest that the 'at risk' limb should be avoided by health care providers, except in life threatening situations (2). Evidence supporting avoidance of the 'at risk' arm is limited due to problems surrounding ethical recruitment to trials. However, a three year follow up study by Clarke et al, involving 188 patients showed that hospital skin puncture was a significant risk factor, with a relative risk of lymphoedema of 2.44 for those who had had skin puncture, versus those without (3).

A paper in the European Journal of Cancer in 1998 reported ten patients whose lymphoedema was thought to have been triggered by, or worsened by venepuncture and cannulation. The paper outlined the important role of healthcare professionals in both educating patients and ensuring patient safety with regards to the condition (4). A case report published in June 2012 described a case of a patient with a history of axillary node dissection 26 years previously, who developed lymphoedema following a routine travel vaccination (5).

A literature review published in 2009 showed rates of lymphoedema ranging for $16-28 \%$ for women who had undergone axillary lymph node dissection, with lower rates after sentinel lymph node biopsy. Evidence suggests that venepuncture can precipitate breast cancer related lymphoedema $(B C R L)$ in patients with axillary surgery due 
to reduced immune function and decreased lymphatic proliferation in response to infection (6).

A prospective study of 1253 women with breast cancer in 2003 showed arm lymphoedema rates of $34 \%$ in patients undergoing axillary lymph node dissection, compared with $6 \%$ in those who only had sentinel lymph node biopsy (7). A second prospective study, published in 2006, found a lymphoedema rate of $7 \%$ amongst 2904 women undergoing sentinel lymph node biopsy (8). As a result of these studies, we will be including patients who have had a sentinel node biopsy in our intervention, as patients are still at risk of developing the condition following this procedure.

\section{Baseline Measurement}

We created an anonymous online survey for staff, which we distributed via the trust-wide email service, with twenty-six responses. Although most staff were aware that procedures should be done on the opposite side in patients with at risk arms, $69.2 \%$ had received no training on lymphoedema prevention. $26.9 \%$ had seen procedures being performed on at risk arms in nonemergency settings. $83.3 \%$ felt that an intervention to make susceptible patients more identifiable to staff would be useful. Ideas for possible interventions included: a patient alert card, wristbands, improved patient education, signs above beds, and coloured stickers on notes.

We also conducted a baseline two-week audit of 12 inpatients undergoing elective axillary surgery for breast cancer. None of these patients had a measure in place to make them more identifiable to ward staff at nine hours post admission.

See supplementary file: Baseline measurements.docx

\section{Design}

We opted for a three-fold intervention. Firstly, we created an 'at risk arm' alert on the computerised patient record, which displays a star next to the patient's name on the virtual ward list. When our trust computer system is updated, this will be more clearly visible to users, and will also include the side which is at risk. We implemented this for new axillary surgery patients, with the plan for future Foundation Year Doctors to add an alert for all patients who are admitted for axillary surgery under our team.

Secondly, we produced pink laminated warning signs to be displayed above the patient's bed when admitted. The signs warn staff that blood pressure, cannulation, venepuncture and injections should be done on a certain side, except in emergencies. The signs are stored in a document wallet at the front of each susceptible patient's notes along with a brief explanation of how and why to utilise the sign. Patient addressograph labels are attached to each sign, to ensure they are returned to the correct patient's set of notes on discharge. The signs can then be re-used whenever the patient is re-admitted within the trust.

We will be applying to the financial department for funding for the signs, to ensure they are sustainable and can be made to meet the ongoing demand. Placing the signs in the front of the notes for each new patient will be part of the Foundation Year Doctors role when preparing other key documentation pre-operatively e.g. drug charts, consent forms.

Finally, we began to include a message to GPs on each discharge summary, informing them that the patient has had axillary surgery and should have procedures done on the opposite side. This hopefully helps to ensure the patient is at a decreased risk of iatrogenic lymphoedema once discharged.

\section{Strategy}

PDSA Cycle One:

We created a small sample of prototype pink signs, for use on the elective surgical ward. We were happy to discover that our signs were being put up above most beds, and were noticed and commented on by several members of staff.

However, due to a shortage of beds on the general surgical wards, some patients were taken to an orthopaedic ward post-operatively, where the usage of signs was lower. This may have been due to the staff on the general surgical wards helping to develop and thus having more information about the intervention.

We also found that some signs were being re-used rather than being returned to individual sets of notes upon discharge. Nursing staff also wanted to know where signs were kept, so that they could place them in the notes when necessary.

We found a drawer on the general surgical ward to store the signs. We also created an information sheet which we sent round in the trust wide email bulletin, to ensure staff looking after axillary surgery patients on other wards are aware of the intervention.

\section{PDSA Cycle Two:}

We created an 'At Risk Arm' alert for a small sample of patients. We found that the current computer system in the trust does not clearly display alerts for all users, instead displaying a star next to the patient's name, without a clear indication as to why the patient has an alert e.g. fall risk, dementia, at risk arm.

We have spoken to the IT Department to ensure that the computerised alert is clearly displayed on each patient's record when the system is updated in six to nine months time.

\section{PDSA Cycle Three:}

We realised that our intervention would not help to protect our patients once they were discharged from hospital. To try and solve this, we began to include a message to the GP on our discharge summaries. However, this was sometimes forgotten about by members of the team.

PDSA Cycle Four: 
We provided the Day Surgery Unit with a supply of signs, to ensure that patients undergoing axillary surgery, but not being admitted to the wards, are still included in the intervention.

PDSA Cycle Five:

After we had implemented our signs on the ward, we discovered that a new 'Admissions on the Day Lounge' was being set up at our hospital. We placed a supply of signs in the Admissions Lounge, and placed signs in notes on the afternoon prior to admission.

We will arrange a brief handover with the new junior doctors in our department, to ensure they are willing to, and aware of, how to implement the interventions. We plan to carry out a further postintervention measurement six to eight weeks after the changeover of jobs. We hope these interventions will become part of the Breast Surgery junior doctors' roles, and that this will help to ensure patient safety and decrease the risk of our patients developing Breast Cancer Related Lymphoedema.

\section{See supplementary file: PDSA Cycles.doc}

\section{Post-Measurement}

We conducted a second anonymous online staff survey after implementation, with 14 responses.

$46.2 \%$ felt that the computerised alerts and $53.8 \%$ felt that the pink signs would aid identification of patients at risk of lymphoedema. $61.5 \%$ felt that susceptible patients being more identifiable to staff would decrease the likelihood of procedures being performed on at risk arms. Staff felt that further interventions such as alert cards and 'At Risk Arm' wristbands would also be useful.

We also carried out a measurement of the number of patients who have an alert sign displayed above their bed. $100 \%$ of eligible patients had a sign displayed above their bed at nine hours post admission.

\section{Lessons and Limitations}

We learnt several important lessons from carrying out our project.

1. We had hoped to contact patients to obtain their views on how prevalent the problem was and ideas about possible interventions. Unfortunately, we were not allowed to contact past patients about the issue, as some felt this may cause patients to believe they had been given sub-optimal care in the past. We found this quite frustrating, as it meant that we were unable to ask patients about which interventions they would find acceptable. We may have designed a more patient-centred intervention e.g. an alert card had we been able to speak to patients about the project. Additionally, we could have created an information sheet for patients to warn them about the side effects of having procedures performed on the side of surgery. This information is usually given to patients by the Breast Care Nurses.
2. Getting key stakeholders engaged is an essential but difficult step in carrying out a quality improvement project. Although most agreed that there was a problem, it sometimes proved hard to engage them further due to the busy nature of their jobs. A few members of staff were also unconvinced about the evidence behind 'at risk arms', making it more difficult to engage them in the project.

3. We found that our computerised patient record system also imposed some limits in terms of computerised intervention. We had ideally wanted to implement an intervention which would display a pop-up message when ordering blood tests on susceptible patients. This would have served as an extra reminder to warn users about which arm to avoid if possible. We discussed this with our IT department, and it was felt that this would not be possible to implement given the current constraints of the software.

4. Informing all staff about the intervention was also tricky. We produced an information sheet which we circulated in the trust wide email bulletin for approximately two weeks. We also spoke to key stakeholders face-to-face to explain the interventions and answer any questions. However, it is highly unlikely that all clinical staff read the email and accompanying information sheet. This was clear in our post-intervention staff survey, where some comments made, for example, about confidentiality, showed that staff were not fully informed about the interventions.

5. We feel that funding and sustainability could potentially be a problem in the future. We paid for and made the prototype signs ourselves. There is a strong possibility that the interventions could fail if no future Foundation Year Doctors take on responsibility for making the signs, ensuring there are good supplies in different departments etc. We also need to agree a policy with our trust to ensure that the signs are financed by the hospital in future.

We rolled out our intervention for current axillary surgery patients at our hospital with mixed success. We had hoped to include previous axillary surgery patients in our intervention. However, obtaining lists of previous patients and then accessing their notes proved too timeconsuming. Additionally, this would have meant producing a much larger number of prototype signs, which would have cost us more time and money.

In order to include previous patients in the interventions, we could ask for a short section to be added to the clerking proformae in the A\&E and pre-assessment departments. This would ascertain whether the patient had had previous axillary surgery, and advise the staff member to add an alert and sign to the notes if this was the case. However, there are potential problems with this strategy, given the time constraints when clerking patients.

Additionally, some current areas of the proforma are often ignored, and it is likely that our section could be deemed as less important than key sections such as drug history, respiratory examination etc. In order to reach more staff, we feel that information about our intervention could be included briefly during staff clinical skills teaching sessions on venepuncture and cannulation. This will involve engaging those in charge of staff teaching sessions, and again, its success will be largely dependent on time constraints. 
Finally, to roll our interventions out further, we will need to secure funding for the signs, and someone who is willing to produce batches of signs when needed. We are discussing this with the finance department at our trust.

\section{Conclusion}

We hope that our intervention will make patients more identifiable to staff, thus decreasing the risk of iatrogenic lymphoedema. With more time and funding, we could also introduce an alert card for all axillary surgery patients, to be shown prior to any potentially harmful procedure being performed.

We encountered problems with contacting patients to obtain feedback on the proposed interventions, as some staff felt this may lead patients to believe they had received sub-standard care in the past. Many staff felt that introducing a coloured wristband would be the best intervention. However, this was not possible due to a trust limit on the number of wristbands allowed per patient.

A new computerised patient record system is due to be implemented in our trust next summer. We are currently in discussion with the designers, to try and ensure that alerts are clearer on the new system. We are also planning on a system whereby an extra blood label will print for susceptible patients, to warn staff taking blood that the patient has an at risk arm.

\section{References}

1. Moffatt, CJ. Lymphoedema: an underestimated health problem. QJM: An International Journal of Medicine. 2003; 96: 731-738

2. Royal College of Anaesthetists. Breast Cancer Related Lymphoedema- Information for Doctors. 2009 August. Available at URL: https://www.rcoa.ac.uk/document-store/breast-cancer-relatedlymphoedema-... [Accessed 21/08/2012]

3. Clarke B, Sitzia J, Harlow W. Incidence and risk of arm oedema following treatment for breast cancer: a three year follow up study. QJM: An International Journal of Medicine. 2005; 98: 343-348

4. Smith J. The practice of venepuncture in lymphoedema. European Journal of Cancer Care. 1998; 7: 97-98

5. Lee TS, Baumgart KW. Vaccines and risk of lymphoedema: a case report of a breast cancer patient. Australian Family Physician. 2012 June; 41(6):404-406

6. Bennett Briton TM, Purushotham AD. Understanding Breast Cancer Related Lymphoedema. Surgeon. 2009 Apr;7 (2):120-4. Review

7. Blanchard DK, Donohue JH, Reynolds C, Grant CS. Relapse and morbidity in patients undergoing sentinel lymph node biopsy alone or with axillary dissection for breast cancer. Archives of Surgery. 2003; 138: 482-488
8. Wilke LG, McCall LM, Posther KE, Whitworth PW, Reintgen DS, Leitch AM et al. Surgical complications associated with sentinel lymph node biopsy: results from a prospective international cooperative group trial. Annals of Surgical Oncology; 13(4): 491-500 